\title{
L'Islamisme, le temps et la femme dans Je viens d'ailleurs de Chahdortt Djavann
}

\author{
Karim Zakaria Nini \\ Université de Montréal
}

La littérature a souvent été l'une des dernières formes d'expression à faire de la résistance face aux nombreux assauts que les sociétés des pays à majorité musulmane ont subis durant le XXe siècle. Si aux débuts du siècle dernier, le colonialisme et l'affirmation identitaire furent parmi les thèmes privilégiés des littératures des pays à majorité musulmane, les préoccupations se réorienteront après les indépendances vers des sujets plus sociaux ou encore politiques tels que la précarité ou la dictature. En Algérie et dans les pays du Maghreb, la littérature fut une arme de premier choix pour les sociétés afin d'exister face aux projets coloniaux d'assimilation ou d'effacement. Parmi les régions à majorité musulmane, le Maghreb est une région particulièrement proche de la francophonie étant donnée la longue et parfois tumultueuse présence française dans des pays comme le Maroc, l'Algérie ou encore la Tunisie.

La littérature dans ces pays s'est naturellement exprimée en français étant donné le besoin de faire face à la présence française, mais aussi de transmettre des messages au « dominant ». La société qui s'exprime dans une langue qui culturellement la marginalise et l'infériorise fait ainsi preuve de maturité. Mais les pays dits du Maghreb ne sont pas les seuls parmi les pays à majorité musulmane à avoir intégré la francophonie dans leur palette culturelle. Des pays comme l'Égypte, le Sénégal, le Liban ou encore l'Iran comptent la langue française dans leurs paysages culturels avec des statuts et une importance qui varient. Dans cet article, j'étudierai le roman Je viens d'ailleurs (2002) de l'écrivaine iranienne francophone Chahdortt Djavann. Issue d'un pays à majorité musulmane, Chahdortt Djavann montre que les littératures de ces pays se sont aussi exprimées, pour diverses raisons, dans la langue de «l'Autre ».

Dans cet article, je montrerai comment la littérature réagit aux bouleversements sociaux qui l'entourent en offrant aux destinataires du discours une critique capable de provoquer les questionnements et les remises en cause. En effet, ma problématique pose la question de la réaction des littératures francophones des pays à majorité musulmane aux bouleversements sociaux et politiques que vivent ces sociétés. Que peut en effet la littérature face aux transformations brutales que peut parfois connaittre une culture? Quel serait par ailleurs le rôle de la francophonie dans la transmission du message au destinataire? Ces questions sont d'autant plus importantes que dans un environnement peut-être peu au fait des cultures de l'islam, des amalgames sont très vite faits et des stéréotypes rapidement 
érigés. Car si la société iranienne, à l'instar de sociétés d'autres pays à majorité musulmane, a connu durant le siècle dernier d'importants conflits culturels et idéologiques qui ont vu l'instauration d'un pouvoir politique d'obédience « islamiste », cette même société a aussi résisté à ce phénomène en déployant divers moyens d'expression afin de montrer la richesse d'un pays et d'une culture appelés à regarder en face leur Histoire et à se réconcilier avec leurs identités multiples.

Dans le roman que j'ai choisi d'étudier, l'écrivaine s'exprime en français pour décrire une réalité qui l'entoure ou qu'elle a connue à un moment ou à un autre de sa vie. De ce fait, elle est la témoin directe d'évènements qu'elle tente de transcrire et de transmettre à un destinataire. Ce destinataire, Chahdortt Djavann l'a voulu francophone. Ce choix je l'explique par le fait que l'écrivaine a voulu par son roman s'adresser à un lectorat plus large que celui limité au sein de ses frontières nationales ou linguistiques. Réputée pour son ouverture et son universalité, la langue française est un véritable instrument de libération pour des écrivains qui se détachent le temps de l'écriture des liens étroits et parfois liberticides qui les lient à leurs communautés, à leurs traditions et à leurs environnements sociaux et politiques où la communication est souvent compliquée. De même, l'écriture en langue française permet à ces écrivains d'offrir leurs témoignages au reste du monde. Ces derniers sont des écrivains que je qualifierais de «lanceurs d'alertes » étant donné la responsabilité qu'ils ont prise d'alerter d'autres cultures des dangers que vivent déjà leurs sociétés. Écrire en langue française c'est refuser de s'isoler au sein de sa culture dont la langue est la frontière. Écrire dans une autre langue que sa langue d'origine est souvent pour les écrivains qui font ce choix, la seule porte vers l'Autre et vers "l'Ailleurs». Mais cela ne signifie pas que Chahdortt Djavann a choisi de se détourner du lectorat iranien non-francophone. Au contraire, en choisissant d'écrire dans une langue autre que celle officiellement et majoritairement adoptée dans son pays, Djavann choisit de s'adresser aux « siens » via un canal plus sûr, « libéré » de la censure potentielle et de l'hégémonie idéologique que subit sa langue d'origine (Reboul 96). Ainsi, en période de crise, l'écrivain tend naturellement à prendre ses responsabilités de «lanceur d'alerte » et d'individu pensant capable de provoquer les questionnements les plus sensibles et les plus importants selon son époque. L'écrivain fait alors de la "littérature engagée », en référence au concept théorisé par Sartre dans Qu'est-ce que la littérature (1948). Mais qu'est-ce qu'un « écrivain engagé? « Pour l'écrivain engagé, écrire revient à poser un acte public dans lequel il engage toute sa responsabilité (rapport entre littérature et monde/société). Il écrit pour son époque, en misant sur sa volonté de rejoindre les hommes et de prendre part aux débats de son présent » (Bruneau 69). Les écrivains des pays à majorité musulmane qui écrivent en langue française visent par ailleurs une plus grande liberté, car selon Sartre : «l'écrivain, homme libre s'adressant à des hommes libres, n'a qu'un seul sujet : la liberté »(Sartre 81). 


\section{Une époque « révolue »}

Chahdortt Djavann est une romancière franco-iranienne. Naturalisée française après avoir fui les autorités au pouvoir après la Révolution iranienne, elle écrit son premier roman en 2002, Je viens d'ailleurs, directement en langue française. Une langue qu'elle ne parlait pas à son arrivée en France. À ce propos, elle écrit :

Cette langue a accueilli mon histoire, mon passé, mon enfance, mes souvenirs et mes blessures. Cette langue m'a accueillie. Elle m'a adoptée. Je l'ai adoptée. Mais, quels que soient nos efforts mutuels, les vingt-quatre ans que j'ai vécus sans elle laisseront à jamais une lacune en moi. Une lacune qui n'est pas un vide. Une lacune remplie de langue persane. Et c'est pour cela qu'il y aura toujours du persan dans mon français. (11)

Donc, la langue française permet à Chahdortt Djavann de faire le récit de sa jeunesse dans l'Iran de la révolution à partir d'un point de vue distancé, bien que cette distance soit relative étant donné que Je viens d'ailleurs est une autobiographie assumée. Dans le français de Djavann, il y a du persan, mais il y a aussi beaucoup d'émotions, de la colère et du vécu. Dans ce premier roman de l'écrivaine, le cadre historique que dépeint le récit est précisément divisé en deux grandes périodes: "Avant la révolution» et "Après la révolution ». Dans le cas de l'Iran, l'islamisme en tant qu'idéologie politique s'est installé dans le pays avec la Révolution iranienne qui a renversé le chah, dernier empereur, et qui a installé la République islamique d'Iran.

Mais il est important avant d'aller plus loin dans l'analyse de définir l'un des concepts principaux de ce propos : l'islamisme. Manifestation politique, sociale et parfois interprétée comme un phénomène religieux, l'islamisme est sans doute un phénomène qui puise dans chacun de ces domaines. L'islamisme est une idéologie politique qui s'est principalement construite, comme les nationalismes du XXe siècle, dans l'opposition à l'Autre, considéré comme envahisseur et étranger. Syncrétisme de différentes idéologies politiques du siècle passé, l'islamisme va emprunter aux unes et aux autres ses principales caractéristiques. Car s'il puise dans les mouvements anticoloniaux leur discours de résistance face à l'hégémonie culturelle et économique du "vainqueur», il emprunte par ailleurs ses prétentions de domination de la société, puis du monde aux nationalismes européens des débuts du XXe siècle. En effet, l'islamisme vise à épurer la société de la présence culturelle occidentale considérée comme un prolongement du colonialisme. Pour cela, les militants islamistes visent une domination de tous les aspects de la vie sociale, des manifestations culturelles jusqu'à la vie estudiantine et universitaire comme l'explique Burgat qui décrit comment le mouvement islamiste en Algérie «s'est orienté vers une stratégie d'entrisme dans les institutions du fondamentalisme d'Etat. » (Burgat 182)

L'islamisme doit être lu dans cet article non comme une religion ou comme une culture, mais comme une idéologie politique au même titre que le communisme à titre d'exemple. Une idéologie politique dont le mot 
d'ordre est «la résurrection des pays musulmans» (Kepel 42). Cette résurrection doit passer par une domination et un contrôle des manifestations sociales jusqu'aux moindres, de la vie culturelle à la pratique de l'espace public. Face à cette volonté de mettre la société sous surveillance et de l'impliquer dans le conflit idéologique qui oppose l'islamisme à l'altérité, la littérature a souvent réagi en déconstruisant le discours dominant, car cette dernière offre un point de vue dépassionné en s'attaquant à l'idéologie non frontalement, mais en empruntant des détours que seule la littérature permet (satire, ironie, parodie, etc.). La Révolution iranienne a donc permis l'avènement d'un pouvoir dont l'idéologie se revendique ouvertement de « l'islam politique ». Dans le roman de Djavann, il y a une rupture brutale dans le cadre temporel du récit. Cette rupture coïncide historiquement avec l'arrivée au pouvoir des partisans de Khomeyni, leader du mouvement islamiste iranien :

À l'époque, à la fin de l'année 1978, on parlait de tout, du communisme, du libéralisme, du capitalisme, du nationalisme, des riches, des pauvres, des prisonniers politiques, du pétrole, de l'économie, de la persécution, de la police secrète, la savak. (15)

L'effervescence qui caractérise la révolution se traduit dans la citation cidessus par une succession de mots qui renvoient chacun aux intérêts d'un groupe social. Mais cette effervescence va laisser place à une sorte de «monothéisme politique » où n'est tolérée qu'une seule pensée : celle de Dieu :

Pour tous ceux qui le soutenaient, le seul parti légitime était le Hezbollah, le « Parti de Dieu » né après la révolution. Un de leurs slogans proclamait : «Un seul parti - Parti de Dieu — Et un seul guide - L'âme de Dieu. (24)

Ce passage traduit les transformations qui ont eu lieu à la suite de la révolution et de l'instauration de la République islamique d'Iran. L'écrivaine, qui a vécu les évènements, dépeint dans son récit la rupture entre deux époques : une époque où il semblait y avoir une certaine liberté d'expression, et une autre où tout le pays devait se tenir en rang derrière un projet « unique » d'inspiration divine. Je viens d'ailleurs est un roman qui témoigne de la transformation radicale de la société et de l'instauration d'une «nouvelle ère ». Dans son roman, Djavann écrit son retour à Téhéran après de nombreuses années et sa rencontre avec une ancienne amie avec qui elle militait avant la révolution, le témoignage de ce personnage est éloquent :

Tu vois, de cette époque il ne me reste plus que quelques deuils. Trois amis sont sortis de prison après de longues années de détention. [...] À leur sortie, [...] Le monde extérieur avait changé, la société, les gens, moi aussi peut-être. Rien n’était plus pareil. Entre eux et le reste du monde, un abîme s'était creusé, ils ne comprenaient plus. [...] C'est inutile de courir après cette époque. Elle est morte et enterrée avec ceux qui l'ont faite. (146)

La Révolution iranienne est une rupture qui semble avoir fait basculer toute une société dans une nouvelle époque. Dans la littérature d'écrivains qui ont vécu ces changements, la société semble se transformer radicalement en un laps de temps relativement court. Dans son roman, Chahdortt 
Djavann décrit comment l'idéologie du nouveau pouvoir politique infiltre l'école :

Notre directrice, une quadragénaire élégante, fut remplacée. Certaines de nos professeurs se sentaient gênées, peut-être même menacées. Peu à peu, leurs attitudes se modifièrent : les jupes s'allongèrent, les maquillages pâlirent, les voix se firent discrètes. D'autres, qui n'avaient jamais été coquettes, se montraient maintenant plus à l'aise. Dans les classes, nous étudiions sans manuels, [...] Apparemment l'histoire que racontaient nos livres ne tenait plus debout et il fallait écrire la vraie histoire, la bonne. (20)

Cet extrait illustre le souci des idéologies politiques conservatrices de modifier l'environnement et de le transformer à leur image. Pour cela, le pouvoir politique investit d'abord les institutions de l'État comme cela a été décrit par Burgat qui appelle cette démarche la «stratégie d'entrisme ». Dans le passage précédent, c'est de la femme, de son intimité et de son libre dont il est question. La « jupe » de la femme est en effet un symbole fort.

Mettre fin aux anciennes pratiques et en instaurer de nouvelles, voilà le moment fondateur, la frontière où prend fin l'ancien monde et où commence le nouveau. Dans son roman, Djavann décrit les moments où l'Histoire du pays a commencé à basculer. L'islamisme au pouvoir est une idéologie de «l'Un » (Zaoui 27). Il n'y a pas de cohabitation possible avec d'autres conceptions du monde et tout l'environnement devait se modeler selon la sienne :

La salle de spectacle avait été transformée en une sorte de mosquée, de lieu de prière. Dans la cour, les murs s'étaient couverts des slogans des différents partis politiques. Les révolutionnaires se distinguaient les uns des autres, dans notre école comme dans le pays, par leurs différences idéologiques et leurs appartenances politiques. Des conflits s’annonçaient. La liberté allait rencontrer bien des problèmes. (21)

\section{Féminité politique}

Le roman de Chahdortt Djavann, Je viens d'ailleurs, montre par ailleurs comment la condition féminine est politisée au profit de l'idéologie politique dominante. Djavann décrit en effet la prise de possession de l'espace public par cette dernière et sa transformation progressive. Dans le roman, Djavann explique comment la position de la femme en société et son comportement ont plié sous le poids de l'uniformisation. Le passage où l'écrivaine traite de l'école où elle était élève est dans ce sens particulièrement intéressant :

Notre directrice, une quadragénaire élégante, fut remplacée. Certaines de nos professeurs se sentaient gênées, peut-être même menacées. Peu à peu, leurs attitudes se modifièrent : les jupes s'allongèrent, les maquillages pâlirent, les voix de firent discrètes.

D'autres qui n'avaient jamais été coquettes, se montraient maintenant plus à l'aise. (20)

Ce passage illustre l'installation progressive de l'idéologie islamiste au sein de la société. La féminité est bien évidemment au centre des préoccupations des nouvelles forces politiques au pouvoir qui s'occupent d'abord de transformer à leur image l'élément le plus visuel et le plus représentatif des valeurs d'une société : la féminité. Dans ce passage, ce n'est pas la femme 
qui est visée, mais la féminité : les jupes, les maquillages et les voix. Par ailleurs, dans cet extrait, d'autres femmes, "moins coquettes », ne voient pas de problèmes à ce que ces éléments soient sous contrôle, car elles se situent en dehors de ces standards. Ces femmes «moins coquettes» ne sont donc pas particulièrement opposées à la libération de la femme, mais se sentent soulagées que l'élément de leur oppression soit aboli : les standards de beauté féminine occidentaux. L'écrivaine décrit en effet ces femmes comme des femmes qui «n'avaient jamais été coquettes». En réalité, ce genre de description renforce le sentiment de frustration chez les femmes dont les standards de féminité sont différents. Cette frustration qui est récupérée par l'islamisme dans ses discours et de laquelle se nourrit cette idéologie. L'écrivaine décrit les autres femmes comme des femmes qui manquent de féminité, de « coquetterie ». Ce sont en réalité des femmes dont les standards de «beauté féminine» sont différents. L'islamisme avant de prendre les apparences d'une idéologie opposée à la féminité est avant tout une idéologie anti-occidentale. En s'attaquant à la « coquetterie », l'islamisme s'attaque à l'un des éléments culturels les plus apparents dans la culture dite occidentale : les standards occidentaux de beauté féminine. Il est ainsi possible que des femmes qui ne se reconnaissent pas dans ces standards de beauté se dressent contre ces mêmes standards et donc contre d'autres femmes, par jalousie, frustration, ou par conviction idéologique ou religieuse. C'est le cas dans le passage précédent où des femmes qui «n'avaient jamais été coquettes» semblent satisfaites du comportement austère que les nouvelles mœurs imposent. C'est aussi le cas dans un autre extrait du roman où le personnage d'une femme tombée enceinte sans être mariée traduit la problématique d'être une femme dans une société emprunte d'islamisme et de traditionalisme. Le personnage de la femme enceinte, une villageoise, est pourchassé par des habitants de son village pour avoir compromis «l'honneur » de son père. Dans le roman, le discours patriarcal est assumé par un personnage féminin :

- Écoutez je ne peux rien faire, et d'ailleurs qui êtes-vous? Vous êtes sa mère?

- Ah! sa mère... Elle est tombée en syncope, dit-elle en hochant la tête. La pauvre femme, tout est fini pour elle, et il n'y a pas qu'elle : son mari a perdu son honneur, il est devenu fou, il voulait tuer la petite. Pauvre famille... ils ne se relèveront jamais d'un tel malheur. (91)

Dans une société encore régie par des traditions conservatrices, la femme n'est pas propriétaire de son corps. Ce dernier est en effet la propriété de la famille et de la société, et la femme a la responsabilité d'en sauvegarder «la pureté ». Le fait que la jeune femme décrite dans la citation précédente soit tombée enceinte en dehors des liens du mariage est considéré dans ce contexte comme le crime ultime qu'une femme peut commettre. Ainsi, le père de la femme incriminée est le premier concerné par cet évènement, car il s'agit de son honneur. Dans des sociétés traditionalistes et patriarcales, l'honneur de la famille dépend de l'honneur du père qui est à son tour intimement lié à la réputation de sa fille ou de ses filles. Une fille qui «perd » 
sa virginité en dehors des liens du mariage commet le crime de transgresser les règles de la communauté. Comme sanction immédiate, celle-ci est frappée de déshonneur ainsi que toute sa famille. Dans le passage précédent, le discours patriarcal qui soumet la vie d'une femme à des enjeux d'honneur, de déshonneur, et de bonne conduite corporelle est repris par une femme. C'est en effet une " grosse femme » qui reprend dans son intervention des éléments du discours patriarcal. En adoptant ce discours, ce personnage l'approuve de facto. Cette scène n'est pas anodine et doit attirer l'attention sur le fait que dans les sociétés durement patriarcales, les femmes se retrouvent souvent complices du traitement auquel est soumise la féminité. Dans ce passage, « une grosse femme » du village revendique fortement des droits sur le corps et la vie d'une jeune fille.

Cette situation rejoint celle de l'extrait où les «femmes qui n'avaient jamais été coquettes » se sentent plus à l'aise dans une société où certains éléments associés à la féminité soient soumis à l'austérité. Cette dernière situation s'explique par le fait que ces femmes qui «n'avaient jamais été coquettes » se sentaient, avant l'installation de l'islamisme, reléguées et confinées en bas de l'échelle de la «beauté féminine » selon les standards de leur société. La transformation de ces standards fait que ces femmes sont évidemment satisfaites des nouvelles mœurs, des mœurs où elles ne sont plus considérées comme «moins coquettes ». Il est ainsi possible d'imaginer que certaines femmes se satisfassent des contraintes qu'impose le nouveau pouvoir politique au statut de la Femme quand ces contraintes les soustraient d'une situation tout aussi violente, celle de devoir se soumettre à des standards de beauté dans lesquels elles ne se reconnaissent pas. Dans le dernier extrait, une villageoise décrite sous des traits négatifs, comme étant une "grosse femme», adopte le discours patriarcal et décrit le fait que la jeune femme soit tombée enceinte comme un malheur. Le roman de Djavann décrit une condition féminine complexe où les femmes, soumises à différentes formes de violence, passent dans certaines situations du côté de «l'oppresseur » pour éviter de se retrouver dans la position de cibles.

Le discours patriarcal n'est pas un discours proprement islamiste. C'est un élément du traditionalisme de la société que l'islamisme récupère afin d'en utiliser la rhétorique. Marc Angenot dans Les idéologies du ressentiment (1996) explique comment la plupart des idéologies du XXe siècle utilisent le ressentiment comme élément fondamental dans leur discours, un ressentiment où « les valeurs que les dominants reconnaissent et prônent sont dévaluées en bloc» (11). Dans le cas des deux extraits précédents, il semble que l'idéologie islamiste utilise la condition féminine difficile dans la société décrite et le ressentiment qui peut en être conséquent pour diviser les femmes et les montrer les unes contre les autres. Le fait que des femmes qui «n'avaient jamais été coquettes» se sentent plus à l'aise dans une société où la féminité est une proie, et qu'une "grosse femme» revendique des droits sur la vie d'une jeune fille sont deux illustrations qui décrivent les deux types de 
violence dont les victimes sont les femmes dans la société décrite : une violence due au système patriarcal traditionnel où le statut de la femme est infériorisé, et une autre qu'imposent des critères de beauté qu'on pourrait décrire comme "occidentaux » et qui instaurent une violente compétition entre les femmes d'une même société. En somme, la violence crée du ressentiment entre les femmes et l'islamisme récupère ce ressentiment pour que celles-ci se retournent contre leur propre condition en attaquant d'autres femmes.

Dans Je viens d'ailleurs, l'apparence des femmes est l'une des priorités du pouvoir politique qui s'est installé après la révolution qui, afin de s'imposer dans l'espace public, doit régir le comportement des personnes qui évoluent au sein de cet espace. Le roman décrivant l'installation progressive de l'islamisme dans la société iranienne, il est alors question du portait physique des personnages féminins avant et après la Révolution iranienne. Dans l'extrait suivant, le même personnage est décrit dans les deux contextes sociopolitiques :

Jolie, vive et très attentive aux autres, Mahsa était la première de la classe. Sa frange raide et ses petits yeux noirs de jais donnaient à son visage un air mutin. Elle avait le plus beau sourire du monde. La regarder était un vrai plaisir [...] (19)

La description du personnage de Mahsa change selon le contexte historique dans lequel le personnage est décrit. Dans la citation précédente, Mahsa est décrite avant la révolution. La citation ci-dessous décrit le même personnage quelques années après la révolution :

Cinq années plus tard $[\ldots]$ une silhouette vaguement familière retint mon regard. [...] c'était bien elle, c'était Mahsa. Elle était voilée, j'étais voilée, nous étions toutes voilées depuis quatre ans. (...) Ses mains et sa voix tremblaient. Ses yeux s'étaient éteints. Elle avait vieilli. Elle me paraissait petite. La voir voilée, sans sa frange raide, faisait tomber en ruine le souvenir que j'avais de son visage espiègle. (56)

L'installation de l'islamisme dans la société iranienne telle que celle-ci est décrite dans le roman transforme l'espace public à son image et cette transformation a pour cible les femmes dont le corps, lieu de conflits idéologiques et de luttes pour la liberté, semble défait. Le corps des femmes ainsi voilé symbolise la victoire du discours patriarcal sur la condition féminine. Chahdortt Djavann décrit dans son roman la transformation des mœurs d'une société sous l'effet de bouleversements politiques, mais aussi culturels. Dans le texte, ces bouleversements se manifestent, entre autres, dans les transformations physique et psychologique des personnages féminins.

\section{Conclusion}

La littérature est un lieu où il est possible de défaire le monde et de le refaire. C'est aussi un lieu où il est possible de remonter les frontières du temps quand celles-ci sont définitivement fermées. Il est possible de lire dans 
Je viens d'ailleurs la nostalgie d'un Iran qui n'existe plus. L'histoire est celle d'amitiés perdues, de souvenirs révolus et de convictions qui se sont éteintes. L'Iran est un pays à l'histoire plusieurs fois millénaire, au carrefour des civilisations et où se sont déroulés les conflits idéologiques les plus cruciaux du siècle dernier. Chahdortt Djavann, dans son roman, traduit l'amère désillusion d'une femme qui a cru dans un Iran où il y aurait de la place pour elle et pour ses rêves. Dans son premier roman, l'écrivaine écrit l'Iran postrévolutionnaire à partir de la francophonie. Elle décrit l'avènement d'une nouvelle époque et l'installation d'un nouveau pouvoir mêlant religion et politique. Chahdortt Djavann décrit en effet la transformation de la société iranienne dans la forme et dans le fond. À quoi est due cette transformation? Qu'est-ce qui fut en action au lendemain de la Révolution iranienne? Les transformations décrites dans Je viens d'ailleurs sont en effet politiques et sociales. Il est par ailleurs possible de lire dans le premier roman de Djavann une construction particulière du cadre temporel du récit. Il est possible d'y constater une rupture nette entre d'un côté un Iran au seuil de la révolution, et de l'autre un Iran postrévolutionnaire marqué par d'importantes transformations. Chahdortt Djavann a voulu faire de son roman un lieu où il serait possible de faire le deuil d'un Iran révolu, mais elle a aussi voulu faire de ce premier roman un témoignage précieux sur son vécu dans l'Iran révolutionnaire. Mais la question qui se pose dans ce genre de productions littéraires est la suivante : que peut réellement nous apprendre une œuvre de fiction, écrite en langue française, sur l'Iran, sur sa société et sur sa révolution? L'analyse de ce roman permet de comprendre ce qui fut à l'œuvre au lendemain de la révolution iranienne à partir d'un point de vue lyrique. Mais surtout, l'analyse de ce roman montre que la nostalgie, le deuil et l'amour sont des émotions qui n'ont pas de langue et qui peuvent ainsi traverser aisément l'univers qui existe entre les cultures persane et francophone. 


\section{Bibliographie}

Djavann, Chahdortt. Je viens d'ailleurs. Autrement, 2002.

Bruneau, Judith Emery. »'La littérature engagée »" Québec français, no. 131, 2003, pp.68-70.

Burgat, François. L'islamisme au Maghreb. Payot, 2008.

Kepel, Gilles. Le Prophète et Pharaon. Gallimard, 2012.

Reboul, Olivier. «Parlons-nous déjà le "Newspeak" d'Orwell? », Autres

Temps : Les cabiers du christianisme social, no.4, 1984, pp. 96-100.

www.persee.fr/doc/chris_0753-2776_1984_num_4_1_987

Sartre, Jean-Paul. Qu'est-ce que la littérature? Folio essais. 1985.

Zaoui, Amin. La Culture du sang. Le Serpent à Plumes, 2003. 